\title{
TEXTURE AND GRAIN SIZE CONTROL DURING ANNEALING OF AN Al-SiC COMPOSITE MATERIAL
}

\author{
D. Juul Jensen and N. Hansen \\ Materials Department, Risø National Laboratory, DK-4000 Roskilde, Denmark
}

\begin{abstract}
The effects of different heat treatments on the recrystallized texture and grain size of $90 \%$ cold rolled aluminium reinforced by 2 vol\% SiC whiskers have been studied. Annealing temperatures in the range $250-510^{\circ} \mathrm{C}$ and 3 heating rates $\left(25,80,400^{\circ} \mathrm{C} / \mathrm{min}\right)$ were used. The recrystallization textures were determined by neutron diffraction and the grain sizes were measured by optical microscopy. It was found that the recrystallization textures are rotated cube textures $(\{100\}<013>$ ) and the strength depends strongly on annealing temperature, $(\mathrm{T})$ and heating rate $(\mathrm{dT} / \mathrm{dt})$ - peak intensities in the range from 6 to $22 \mathrm{x}$ random are measured. Also the grain size varies strongly (70-435 $\mu \mathrm{m}$ ) with $\mathrm{T}$ and $\mathrm{dT} / \mathrm{dt}$. Simple maps relating the effects of $\mathrm{T}$ and $\mathrm{dT} / \mathrm{dt}$ on texture and microstructure are constructed and it is discussed how $\mathrm{T}$ and $\mathrm{dT} / \mathrm{dt}$ affects nucleation and growth.
\end{abstract}

\section{INTRODUCTION}

An important scientific as well as practical aim is to be able to model and control the evolution of texture and grain size during thermomechanical processing ${ }^{1,2}$. For a given material, this requires knowledge about the effect of a whole range of process parameters such as strain, strain rate, temperature and heating rate. As the effects of these different process parameters are not independent, it is indeed not straightforward to predict the resulting textures and grain sizes. Experiments covering a very large parameter range are in general needed. In the present work, the effects of variations in the annealing temperature $(\mathrm{T})$ and heating rate $(\mathrm{dT} / \mathrm{dt})$ have been studied for static recrystallization of heavily cold rolled $\mathrm{Al}+2 \mathrm{vol} \% \mathrm{SiC}$ whiskers. This material was chosen because it belongs to the class of new metal matrix composites reinforced with short fibres or particles which are emerging as potential engineering materials and because earlier investigations ${ }^{3,4}$ have shown that the annealing temperature has a remarkable large effect on the recrystallization texture and grain size. As a result of the present investigation, maps relating the effects of $\mathrm{T}$ and $\mathrm{dT} / \mathrm{dt}$ on texture and grain size are constructed. 


\section{EXPERIMENTAL}

Al powder containing 0.8 vol\% $\mathrm{Al}_{2} \mathrm{O}_{3}$ and 2 vol\% $\mathrm{SiC}$ whiskers (supplied by Tokai Carbon Co.) of $\beta$-type cubic single crystals, 0.1-1 $\mu \mathrm{m}$ in diameter and 30-100 $\mu \mathrm{m}$ in length, were blended. After consolidation about $40 \%$ of the whiskers are rather uniformly distributed in the $\mathrm{Al}$ matrix, but about $60 \%$ are still present in clusters with size ranging from 0.5 to $5 \mu \mathrm{m}$. During the processing the whiskers are fragmented and on average their length is reduced to $2 \mu \mathrm{m}$. For further details see Liu, Hansen and Juul Jensen ${ }^{3}$.

The starting material was cold rolled to $90 \%$ reduction in thickness. The annealing of the specimens was done isothermally in 3 types of furnaces: salt bath, mini furnace and standard furnace. As a result heating rates, dT/dt equal to $400^{\circ} \mathrm{C} / \mathrm{min}, 80^{\circ} \mathrm{C} / \mathrm{min}$ and $25^{\circ} \mathrm{C} / \mathrm{min}$ were obtained. Annealing temperatures in the range 250 to $510^{\circ} \mathrm{C}$ were used. The recrystallization textures were measured by neutron diffraction. ODFs were calculated using the series expansion method, $\lambda \leq 22$ and only even $\lambda$ terms. The recrystallized grain sizes were measured in the rolling plane section by optical microscopy using a semiautomatic computerized image analyzer.

\section{RESULTS}

\section{Texture.}

The dominant component in the recrystallization texture is the $\mathrm{N}$ component $(\{100\}<013>)$. (The ODF is shown elsewhere in these proceedings $\left.{ }^{5}\right)$. Besides this component only weak peaks (intensities below $5 \mathrm{x}$ random) are observed. A detailed discussion of the origin of this texture is also given elsewhere in these proceedings ${ }^{5}$. For all $\mathrm{T}$ and dT/dt's, $\mathrm{N}$ was found to be the main component; however $\mathrm{T}$ and $\mathrm{dT} / \mathrm{dt}$ affects the development of the weak components. For example, for salt bath annealing (dT/dt $=400^{\circ} \mathrm{C} / \mathrm{min}$ ) retained rolling components were observed for low temperature annealing $\left(\mathrm{T}<350^{\circ} \mathrm{C}\right)$ whereas components near $\{110\}<332>$ were observed for annealing at higher temperatures.

The strength of the $\mathbf{N}$ component depends strongly on $\mathrm{T}$ and $\mathrm{dT} / \mathrm{dt}$. This is illustrated in Fig. 1a. In the figure results for the highest and lowest heating rates are shown. For $\mathrm{dT} / \mathrm{dt}=400^{\circ} \mathrm{C} / \mathrm{min}$ the texture strengthens with increasing temperature, whereas the opposite is the case for $\mathrm{dT} / \mathrm{dt}=25^{\circ} \mathrm{C} / \mathrm{min}$. These data (together with the data for $\mathrm{dT} / \mathrm{dt}=80^{\circ} \mathrm{C} / \mathrm{min}$ ) may also be presented in the form of a map showing the strength of the $\mathrm{N}$ component as functions of $\mathrm{T}$ and $\mathrm{dT} / \mathrm{dt}$ (see Fig. 2a). A low annealing temperature and low heating rate results in a strong $N$ texture $(>20 \mathrm{x}$ random). If $\mathrm{T}$ or $\mathrm{dT} / \mathrm{dt}$ is increased the texture weakens toward a minimum in the intermediate region (high $\mathrm{T}$ and low dT/dt or low $\mathrm{T}$ and high $\mathrm{dT} / \mathrm{dt}$ ). Here the $\mathrm{N}$ texture is below $10 \mathrm{x}$ random. In the high region (high $\mathrm{T}$ and high $\mathrm{dT} / \mathrm{dt}$ ) the texture strengthens and intensities above $10 \mathrm{x}$ random (but below $20 \mathrm{x}$ random) are measured.

\section{Grain Size.}

The recrystallized grains were inspected in the rolling plane. In this plane the grains are equiaxed and the distributions fairly homogeneous. The mean grain size depends on the annealing parameters T and dT/dt. This is illustrated in Fig. 1b, and Fig. 
$2 \mathrm{~b}$. The figures show that when the annealing temperature is increased the grain size decreases. The largest effect is observed for the lowest heating rate, $\mathrm{dT} / \mathrm{dt}=25^{\circ} \mathrm{C} / \mathrm{min}$, here the average grain size is reduced from $435 \mu \mathrm{m}$ to $92 \mu \mathrm{m}$ when $\mathrm{T}$ is increased from $310^{\circ} \mathrm{C}$ to $420^{\circ} \mathrm{C}$.

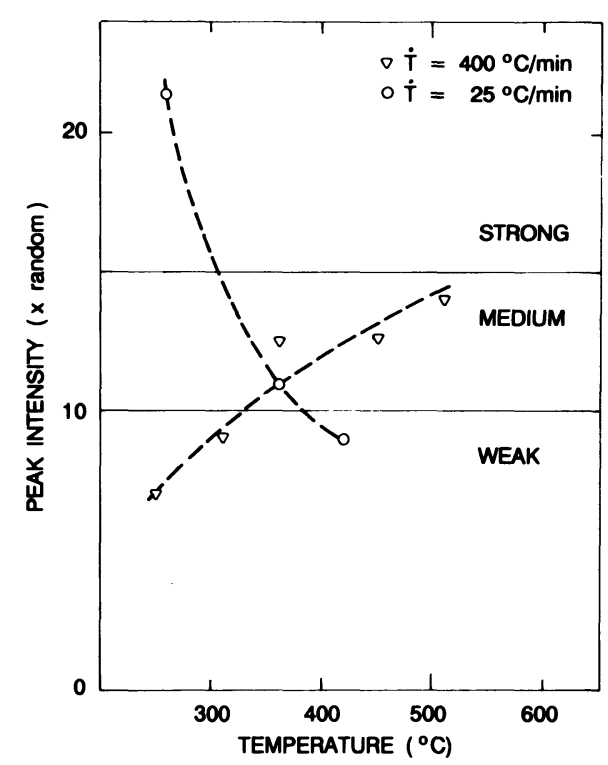

a)

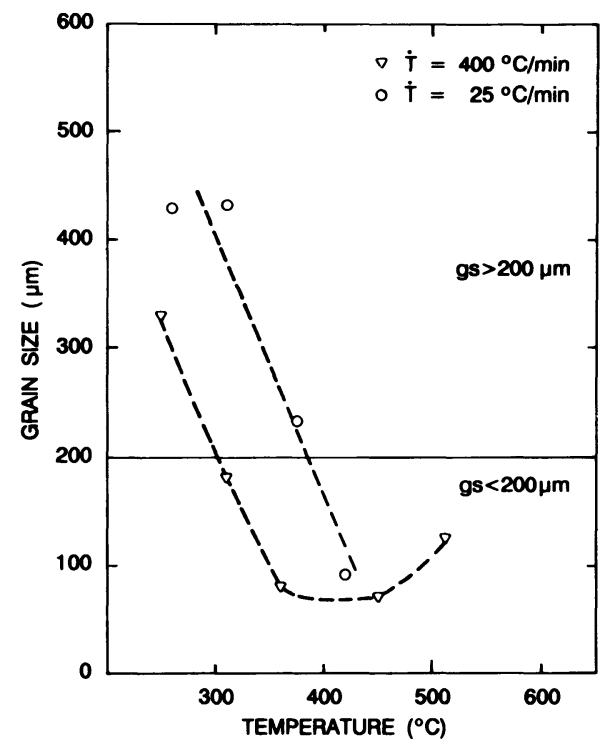

b)

Fig. 1. The effect of annealing temperature and heating rate $(\dot{T})$ on a) the peak intensity of the $\mathbf{N}$ component and $\mathrm{b}$ ) the mean recrystallized grain size (equivalent circle diameter) in the rolling plane.

\section{DISCUSSION}

The two heat treatment parameters, the heating rate $\mathrm{dT} / \mathrm{dt}$ and the annealing temperature $T$, were found to have very significant effects on the recrystallization texture and grain size. Data of this type may be used to get information about the nucleation and growth processes as well as, of more practically importance, to chose process conditions which give optimal recrystallized materials. A detailed discussion of the effects of $\mathrm{T}$ and $\mathrm{dT} / \mathrm{dt}$ on the nucleation and growth for each annealing condition is beyond the scope of this short article and would require further investigations of the relations between texture and microstructure for example using the electron back scattering technique (EBSP) ${ }^{6}$. Thus, in the following earlier results ${ }^{4}$ for the effect of $T$ on nucleation and growth will be used to give a qualitative description of the combined effects of T and dT/dt. Furthermore it will be discussed how texture/grain size maps (as shown in Fig. 2) may be used to tailor the conditions of the recrystallized material.

Microstructural investigations ${ }^{3}$ have shown that the principal nucleation sites are clusters of $\mathrm{SiC}$ whiskers, single $\mathrm{SiC}$ whiskers, other large particles $(\mathrm{FeAl} 3)$ and original grain boundaries (see Table 1). The orientation of the nuclei developed at these 


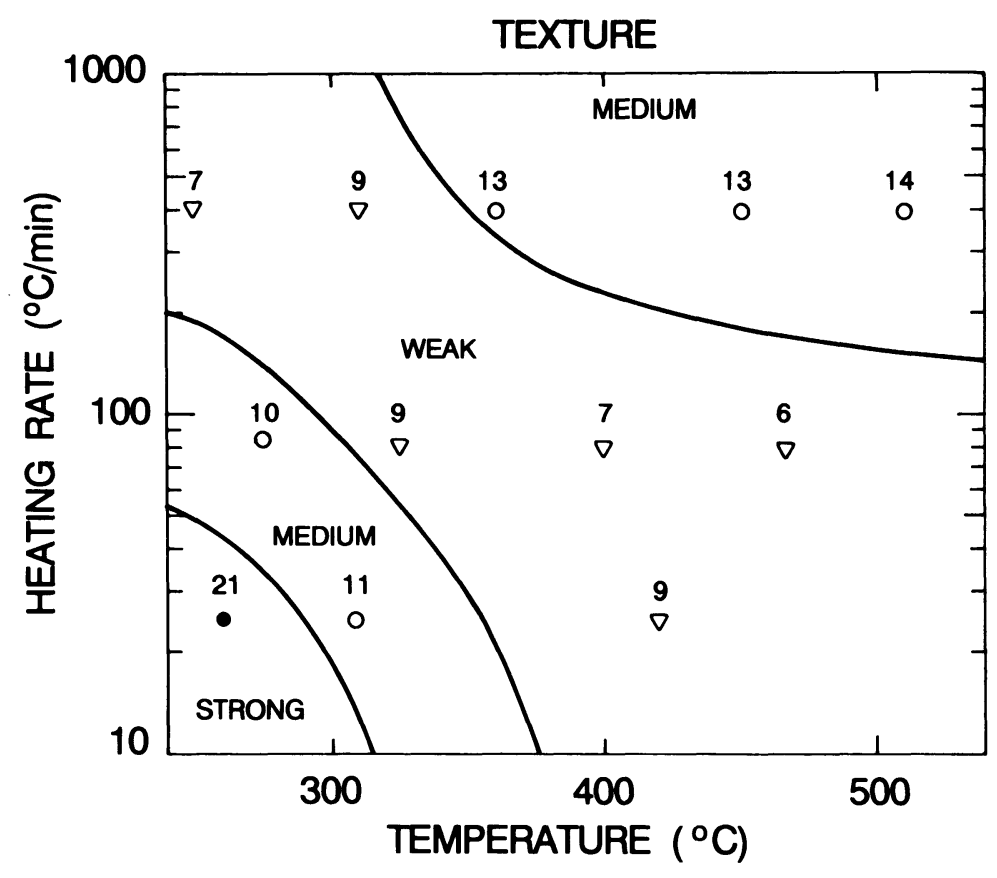

a)

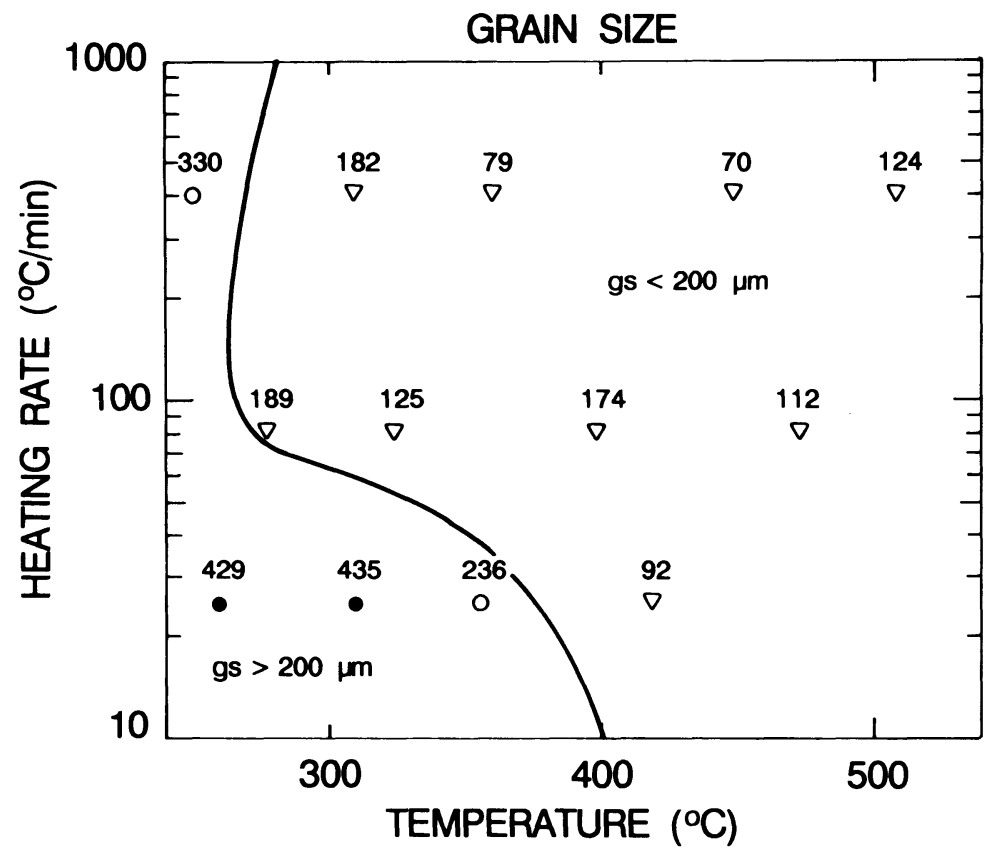

b)

Fig. 2. Maps showing the effect of annealing temperature and heating rate on texture and microstructure. a) strength of $\mathrm{N}$ texture. b) mean recrystallized grain size. 
different sites depend on the type of site and can be classified as follows. SiC clusters may give $\mathrm{N}$ nuclei if they are situated in a deformed matrix region with orientations not too far from $\{100\}<013>$; otherwise the SiC clusters give nuclei of other orientations, here referred to as $\mathrm{O}$ nuclei. Also single $\mathrm{SiC}$ whiskers and other large particles may result in spread rolling or $\mathbf{O}$ nuclei. Finally, the original grain boundaries are assumed to give nuclei of retained rolling orientations, however, as only weak retained rolling texture are observed in the present series of experiments no special emphasis will be on this component and in the following it will be included in the O-texture.

For heat treatments using a high heating rate and a high annealing temperature, all types of nucleation sites may become active, i.e. many nuclei develop resulting in a fairly small recrystallized grain size (see Table 1). Only few of the nuclei are of the $\mathrm{N}$ orientation, but the recrystallization texture not only reflects the orientation of the nuclei, also their possibilities of growth. EBSP investigations ${ }^{4}$ have shown that under these annealing conditions many of the $\mathbf{O}$ grains only grow very little, whereas the $\mathbf{N}$ grains are large in the recrystallized state. A medium strong $\mathrm{N}$ recrystallization texture therefore evolves. If the annealing temperature is decreased, the grain size increases, expressing that fewer nuclei develop at the most powerful sites. In this case the recrystallized grain size distributions for the $\mathrm{N}$ and $\mathrm{O}$ grains are almost identical ${ }^{4}$, i.e. the strength of the $\mathbf{N}$ texture decreases. This is also the case if the annealing instead was done at a low heating rate and a high temperature. The present results, however, show that when $\mathrm{dT} / \mathrm{dt}$ is low and $\mathrm{T}$ is high the recrystallized grain size is smaller than that when $\mathrm{dT} / \mathrm{dt}$ is high and $\mathrm{T}$ is low. An explanation may relate to different recovery mechanisms ${ }^{7}$ in the two conditions, however, further investigations are needed to clarify this point. Finally, for a low heating rate and a low temperature, only the very best nucleation sites become active and a large grain size is observed. Under these conditions, also growth is fairly problematic and broad grain size distributions of $\mathbf{N}$ and $\mathrm{O}$ grains are produced. On average the $\mathrm{N}$ grains become larger than the $\mathrm{O}$ grains and a strong $\mathbf{N}$ texture is produced (see Table 1 ).

Table 1. Effect of annealing temperature (T) and heating rate (dT/dt) on nucleation, recrystallized grain size and texture. (" + + " refers to many, " + " to few and "-" to almost no nuclei of a given type).

\begin{tabular}{|lc|cc|cc|}
\hline Nucleation: & & \multicolumn{2}{|c|}{$\mathrm{dT} / \mathrm{dt}$ high } & \multicolumn{2}{c|}{$\mathrm{dT} / \mathrm{dt}$ low } \\
Nucleation site & Orientation & T high & T low & T high & T low \\
\hline SiC cluster & $\mathrm{N}$ & ++ & ++ & ++ & + \\
SiC cluster (other) & $\mathrm{O}$ & ++ & ++ & ++ & + \\
single SiC & $\mathrm{O}$ & ++ & + & + & - \\
large particles & $\mathrm{O}$ & ++ & + & + & - \\
grain boundaries & 0 & ++ & + & + & - \\
Grain size: & & $<300 \mu \mathrm{m}$ & $>300 \mu \mathrm{m}$ & $<300 \mu \mathrm{m}$ & $>300 \mu \mathrm{m}$ \\
$\mathrm{N}$ texture: & & medium & weak & weak & strong \\
\hline
\end{tabular}

The above discussion illustrates the fairly complex effects of $T$ and $\mathrm{dT} / \mathrm{dt}$ on nucleation and growth and therefore on the recrystallization texture and grain size. 
More straightforward information about how to chose annealing conditions to give specific textures and microstructures in the recrystallized state can be obtained from maps as those shown in Fig. 2. If, for example, a material with a small grain size and a weak texture is wanted, a high annealing temperature $\left(T>400^{\circ} \mathrm{C}\right)$ and a low heating rate $\left(\mathrm{dT} / \mathrm{dt}<200^{\circ} \mathrm{C} / \mathrm{min}\right)$ should be used. Or if a large grain size and a strong texture is the optimum, both $\mathrm{T}$ and $\mathrm{dT} / \mathrm{dt}$ should be low (e.g. $\mathrm{T}<300^{\circ} \mathrm{C}$ and $\mathrm{dT} / \mathrm{dt}<50^{\circ} \mathrm{C} / \mathrm{min}$ ).

For predictions outside the original parameter range the maps may also be used at least to give crude guidelines. In the present series of experiment, an annealing using $\mathrm{dT} / \mathrm{dt}=3.3^{\circ} \mathrm{C} / \mathrm{min}$ and $\mathrm{T}=400^{\circ} \mathrm{C}$ was also tried. According to the maps, a grain size just above $200 \mu \mathrm{m}$ and an $\mathrm{N}$-texture about $10 \mathrm{x}$ random should be expected. Experimentally the grain size was determined to $242 \mu \mathrm{m}$ and the texture to $11.2 \mathrm{x}$ random; i.e. reasonable agreement between the predictions and the experiments is in this case obtained.

\section{CONCLUSIONS}

The heating rate (dT/dt) and the annealing temperature $(\mathrm{T})$ have significant effects on the recrystallized texture and grain size in $90 \%$ cold rolled $\mathrm{Al}+2$ vol\% SiC. Even small variations in $\mathrm{dT} / \mathrm{dt}$ and $\mathrm{T}$ may cause large changes in the resulting texture and microstructure. Many experiments are needed to establish qualitative correlations (maps) between T, dT/dt, grain size and texture, which may be used as guidelines for optimization of the recrystallized material. To understand the underlying nucleation and growth mechanisms, relations between nuclei position and orientation as well as relations between grain size and orientation have to be determined by local texture measurements for series of partly and fully recrystallized samples.

\section{REFERENCES}

1. E. Nes and W.B. Hutchinson, In "Materials Architecture" (Bilde-Sørensen et al. eds., Risø 1989) p. 233.

2. A.K. Sachdev and J.D. Embury eds., "Formability and Metallurgical Structures" (The Metallurgical Society AIME 1986).

3. Y.L. Liu, N. Hansen and D. Juul Jensen, Metall. Trans. A, 20A, 1743 (1989).

4. D. Juul Jensen, N. Hansen and Y.L. Liu, Mater. Sci. Techn. (in press).

5. N. Hansen, D. Juul Jensen and Y.L. Liu, These proceedings.

6. D.J. Dingley, Scan Electron Microscopy, 11, 569 (1984).

7. "ALUMINIUM, Properties, Physical Metallurgy and Phase Diagrams" (K.R. Van Horn ed., ASM, Ohio 1967). 